\title{
POBREZA Y DESIGUALDADES SOCIALES: UN DEBATE OBLIGATORIO EN SALUD ORAL
}

\begin{abstract}
César Ernesto Abadía Barrero*
Resumen: Las reformas al sector salud de las últimas décadas en Latinoamérica están fomentando la privatización de los servicios siguiendo principios de corte liberal. A diferencia de un sistema de medicina social, en sistemas privados el acceso de las personas a los servicios depende de la capacidad adquisitiva y su énfasis es más terapéutico que preventivo. El resultado es que personas con más recursos tienen menos riesgo de enfermar y de que sus enfermedades se agraven. De otro lado, quienes viven en la pobreza no solamente son más vulnerables a padecer enfermedades, sino que tienen menos acceso a los servicios de salud y, al no recibir tratamientos adecuados y oportunos, sus enfermedades se agravan. Esta situación es peor aún en temas de salud oral, donde se requiere un debate con la bioética como interlocutora para proponer sistemas de equidad.
\end{abstract}

Palabras clave: salud oral, pobreza, desigualdad, bioética.

\section{POVERTY AND INEQUALITIES: AN OBLIGATORY DENTAL HEALTH DEBATE}

\begin{abstract}
Latinamerican last decades' health reforms have encouraged liberal principles where access to services depend on purchasing power, and where emphasis is placed more on therapeutics than on prevention. Therefore, people with high income present less sickness risks while those with low income are more vulnerable to illness due to their poor access to health services. This situation is even worse in oral health issues; that's why bioethics is urgently required to act as speaker in a debate on equity.
\end{abstract}

Key words: oral health, poverty, inequality, bioethics

\section{POBREZA E DESIGUALDES SOCIAIS; UM DEBATE OBRIGATORIO EM SAUDE ORAL}

Resumo: As reformas do setor saúde das últimas décadas na América Latina estão fomentando a privatização dos serviços, seguindo princípios de cunho liberal. Diferentemente de um sistema de medicina social, em sistemas privados, o acesso das pessoas aos serviços depende da capacidade de aquisiçãoe sua ênfase é mais terapêutica que preventiva. $\mathrm{O}$ resultado é que as pessoas com mais recursos tem menos risco de ficarem doentes e de que suas enfermidades se agravem. Por outro lado, as pessoas que vivem na pobreza, não somente são mais vulneráveis em sofrer enfermidades, mas também têm menos acesso aos serviços de saúde e, ao não receber tratamentos adequados e oportunos, suas enfermidades se agravam com o tempo. Esta situação se encontra agravada em temas de saúde oral, onde se exige um debate com a bioética como interlocutora para propor sistemas de equidade.

Palavras chave: saúde oral, pobreza, desigualdade social, bioética

Oral Health Department. Harvard University. United States

Correspondencia: cesar_abadia@post.harvard.edu 


\section{Introducción}

Los niveles de pobreza de la región latinoamericana, históricamente alarmantes, continúan en aumento en vez de disminuir. En 1980, 41\% de los latinoamericanos vivían en condiciones de pobreza. En 2002, el porcentaje de pobres ya alcanzaba el 44\%, 221 millones de personas; 97 millones de ellos (19,4\% de la población) viviendo en la pobreza extrema o la indigencia(1). Tales niveles de pobreza están asociados a la inmensa desigualdad social de la región, la mayor del mundo. Desigualdad social significa que minorías ricas concentran la mayoría de los recursos del país, mientras que la gran mayoría tiene muy poco. Para disminuir estas desigualdades se requiere, lógicamente, de medidas distributivas que otorguen más ingresos a los pobres y menos a los ricos. Desafortunadamente, en la región latinoamericana los ricos no sólo concentran más recursos sino que reciben proporcionalmente más ingresos que los pobres y, como resultado, se observa que no sólo la pobreza sino también la brecha entre ricos y pobres se ha incrementado en las últimas décadas.

¿Cuál es la relación entre desigualdades sociales y salud? La salud es un proceso complejo, representado por una interdependencia entre factores individuales y sociales y que, por tanto, los procesos salud-enfermedad no dependen exclusivamente de las personas, sino de las interacciones entre la persona y su entorno. Éste favorece la aparición de enfermedades y dificulta o facilita que la persona enferma reciba tratamientos adecuados, controle su enfermedad y se recupere. Personas con más recursos y mejores condiciones de vida tienen menos posibilidades de enfermarse y, en contraste, personas con menos recursos y peores condiciones de vida tienen más posibilidades de enfermarse. Los sistemas de salud y las políticas públicas sociales influyen sustancialmente en este vínculo entre desigualdades sociales y sa- lud. Si destinan más recursos y servicios a los pobres, se pueden compensar los efectos de la pobreza y disminuir las desigualdades sociales. Por otro lado, cuando los sistemas de salud y las políticas públicas disminuyen subsidios sociales y crean barreras económicas para el acceso a los servicios de salud, aumentan las desigualdades sociales.

Los sistemas de salud que prestan especial atención a la relación entre condiciones de vida y salud enfatizan los programas y servicios de promoción y prevención, promueven medidas de saneamiento básico y desarrollo comunitario, y priorizan el tratamiento en un sistema interconectado de servicios con varios niveles de atención según la complejidad de la enfermedad. Este tipo de sistemas, basado en las necesidades de las personas y las comunidades, tienen una gran trayectoria académica y práctica en la región latinoamericana, con varias vertientes de un movimiento conocido como medicina social, medicina comunitaria o medicina colectiva(2). Por lo general, la medicina social se basa en sistemas públicos de salud, los cuales, mediante recolección de impuestos, ofrecen acceso igualitario a servicios de prevención, promoción, tratamiento y rehabilitación en salud a toda la población. Su nacimiento se debe a los sistemas de bienestar social europeos, pero se desarrollaron como un movimiento social latinoamericano desde la década de los setenta, oponiéndose a las muchas dictaduras de la región y a gobiernos elegidos por voto popular que han fomentado las desigualdades sociales e impactado negativamente en la salud de la población debido a manejos económicos y políticas públicas inadecuadas(2).

En el otro extremo se encuentran sistemas que podríamos denominar como "basados en el mercado" o "de pensamiento liberal", los cuales consideran que los servicios de salud deben concebirse como mercancías de consu- 
mo privado, es decir, basadas en las leyes de la oferta y la demanda. Por lo tanto, responsabilizan a cada persona por sus cuidados y situación de salud y por la búsqueda de servicios para su tratamiento. Este tipo de sistemas, basado en los mercados, no contempla respuestas frente a los factores sociales de los procesos salud-enfermedad ni reconoce necesidades específicas en salud de poblaciones más vulnerables a las enfermedades; por el contrario, refleja y afianza desigualdades sociales de raza, género y condición socioeconómica(3).

En la región latinoamericana las reformas del sector salud de las últimas décadas -siguiendo las políticas y presiones de organismos financieros internacionales, en especial del Banco Mundial-están desmantelando los sistemas públicos de salud y fomentando la privatización de los servicios. Estas reformas -que siguen principios de pensamiento liberal (ahora revitalizado en el modelo conocido como neoliberal)- han traído resultados muy negativos a la región, con varios países mostrando retrocesos significativos en indicadores de salud pública(4,5). El informe del Banco Mundial de 1993 "Invirtiendo en salud"(6) abrió la posibilidad de que el modelo de salud privatizante de los Estados Unidos, conocido como atención gerenciada (managed care), fuera exportado a la región latinoamericana, una vez que las compañías multinacionales de seguros en salud vieron que su mercado en ese país se había saturado $(7,8)$.

Ante este panorama de pobreza, desigualdades sociales y sistemas de salud surgen varias preguntas para la profesión odontológica/ estomatológica. ¿Cuál ha sido el aporte de la odontología frente a este panorama de pobreza y desigualdad social en Latinoamérica y el Caribe? ¿Cuáles son las respuestas que la profesión ha brindado frente a esta realidad y cuáles son las estrategias que planean para enfrentar este panorama? ¿Cómo la tradición odontológica ve este debate entre medicina social y medicina basada en los mercados? En este ensayo, más que traer respuestas a estas preguntas, pretendo plantear algunos puntos de discusión que espero nos permitan implementar y desarrollar líneas de reflexión e investigación, y comenzar a generar respuestas frente a la alarmante situación de pobreza y desigualdad social, y a la grave situación de salud oral de la mayoría de la población latinoamericana y caribeña.

Debo aclarar que cuando hablo de "odontología" no me refiero exclusivamente a sus practicantes, sino a una actividad construida históricamente y de la cual hacen parte sus practicantes (profesionales de la odontología), la población en general, los gobiernos -a través de sus leyes y programas sociales en salud-y los mercados nacionales e internacionales, representados por seguros privados y por compañías farmacéuticas, cosméticas, biotecnológicas y de materiales dentales. Por tanto, este ensayo pretende que redimensionemos la profesión odontológica como esta amalgama de actores y acciones que interactúan en un momento histórico en el que compiten dos paradigmas en salud en la región latinoamericana: salud como derecho humano fundamental y universal de la vertiente de la medicina social, y salud como mercancía individual de consumo y supeditada a las leyes del mercado de la vertiente liberal.

\section{Protocolos de manejo. ¿Cómo manejan la odontología y la medicina las desigualdades sociales?}

En medicina, los parámetros de tratamiento están claramente definidos desde el punto de vista biológico y los protocolos de manejo clínico buscan minimizar las posibilidades de error humano. Según los protocolos, los profesionales de la medicina hacen una evaluación de sig- 
nos y síntomas y, apoyándose en medios diagnósticos, deben establecer pautas terapéuticas iguales a todas las personas que padecen de una misma condición, independientemente de su raza, afiliación religiosa, lugar de procedencia o capacidad de pago. Los protocolos buscan que los parámetros de excelencia sean únicos y el manejo y seguimiento de esos parámetros se relacionan con las competencias que la sociedad occidental ha impuesto en el ejercicio de los profesionales de la medicina(9). Sin embargo, en tal búsqueda de perfeccionamiento, los médicos pierden la libertad de negociar su práctica de forma más individualizada y, a pesar de que consideren que otras alternativas de manejo sean mejores que los protocolos, ellos pueden ser castigados si no siguen parámetros estrictos(10). En la práctica clínica no hay posibilidad de ensayar o individualizar el tratamiento, y los ensayos hacen parte de las investigaciones que terminan formulando, con el tiempo, nuevos protocolos.

Los protocolos de manejo clínico, por tanto, se ubican en una negociación intermedia difícil, en la cual se restringen las libertades médicas para realizar tratamientos más individualizados, pero se busca que todas las personas sean tratadas según los parámetros que se consideran como mejores para cada condición/patología. Cabe resaltar, también, que tal énfasis en el mejor tratamiento representa un sesgo hacia un tipo de conocimiento específico que le da más peso a los tratamientos en vez de a la prevención y a los resultados biológicos medibles y reproducibles, es decir, una corriente de pensamiento positivista que se ha afianzado en la biomedicina con la medicina basada en la evidencia (MBE). Este método científico, lejos de ser la panacea que muchos consideran, hace parte de un esquema de negociación y percepción del mundo que, a través de intereses comerciales de industrias privadas, como la farmacéutica, biotecnológica y de seguros, está dictaminando el accionar de la medicina basado más en los intereses de grupos económicos que en las realidades y necesidades de los procesos salud-enfermedad o del bienestar de las comunidades(11-14). Los reportes de la MBE dejaron de ser confiables ya que se sabe que estos poderes económicos en salud (siendo la industria farmacéutica el sector más estudiado, empero, recientemente) sesgan y manipulan los resultados de los reportes publicados, incluso en las revistas científicas más prestigiosas, y coartan la libertad de los investigadores y centros universitarios al financiarles las investigaciones, todo con el fin de mostrar resultados que aumenten el lucro que trae la venta de sus productos $(13,15)$.

En sistemas de servicios de salud que siguen las leyes del mercado es posible entender cómo la condición socioeconómica del paciente determina el tratamiento que recibe. Es decir, un paciente sin capacidad de pago no recibirá tratamientos que no pueda pagar, a pesar de que estos tratamientos sean los indicados en el protocolo clínico. Sin embargo, mantener parámetros de calidad altos es importante, ya que calidad y mercados van de la mano. La tensión entre brindar el tratamiento que la persona necesita y asegurar que el cliente pague por los servicios recibidos se soluciona en la medicina de los mercados dividiendo la atención del pago. Los profesionales deben aplicar los protocolos de manejo clínico de forma igualitaria a todas las personas, y los departamentos de cobro se encargan de que la persona pague por sus tratamientos recibidos, bien sea mediante su póliza privada de seguro, mediante seguros subsidiados con fondos públicos, a través de planes de financiación individual cuando se carece de póliza privada o seguro público, o, en algunos casos, mediante fondos para tratamientos gratuitos. Entonces, el protocolo busca solucionar los problemas que el mercado (la capacidad de pago del paciente) puede traer frente a la calidad de los servicios ofrecidos. 
A pesar de la pérdida de libertad en la práctica clínica, los protocolos pueden ser benéficos si pensamos que a un paciente con un cuadro específico se le debe brindar el mejor tratamiento disponible, independientemente de su raza, condición socioeconómica o lugar de procedencia. Sin embargo, estudios recientes en Estados Unidos muestran que, a pesar de los protocolos, diferencias raciales se traducen en un tipo de discriminación por medio de la cual profesionales de salud ofrecen tratamientos inadecuados o menos alternativas terapéuticas a personas que pertenecen a minorías raciales, aun después de controlar por situación socioeconómica, diagnóstico y tipo de seguro de salud(16). Es decir, dos pacientes, uno de raza negra y otro de raza blanca, con el mismo cuadro clínico, género, edad y con la misma capacidad de pago, reciben en Estados Unidos tratamientos diferentes, uno considerado inadecuado y otro adecuado. Esto se explica analizando la cultura de la salud en Occidente y las relaciones entre desigualdades sociales, razas y privilegios, las cuales han producido una mirada sesgada en los profesionales de la salud que los lleva a ofrecer tratamientos inadecuados a personas de minorías raciales(17).

¿Presenta la odontología un sesgo similar en cuanto a raza y condición socioeconómica? Un estudio con minorías raciales en Estados Unidos (afroamericanos y latinos) parece señalar que este es el caso. En este estudio se encontró que la combinación entre condición socioeconómica y raza hace que los odontólogos ofrezcan menos alternativas de tratamiento a pacientes de minorías raciales comparados con pacientes blancos. A pesar de presentar el mismo grado de severidad y avance en sus patologías, los odontólogos les presentan a personas de minorías raciales menos opciones terapéuticas y sugieren las extracciones como tratamiento en vez de otras alternativas que sí les ofrecen a pacientes blancos(18). Este estudio sugiere que el sesgo médico frente a la raza y la condición socioeconómica también se presenta en la odontología. Sin embargo, en la sección siguiente quiero examinar los protocolos de manejo clínico que se aplican en esta especialidad y responder si la práctica odontológica tiene parámetros universales similares a los de la medicina o sugiere que cada tratamiento debe ser individualizado.

\section{La odontología como práctica que fomenta las desigualdades sociales}

¿Cuáles son las respuestas que, como profesión de la salud, ofrecemos a quienes viven en la pobreza? La práctica odontológica consiste en actividades diagnósticas y terapéuticas que se realizan, por lo general, en consultas privadas y consultas públicas, o en servicios de seguridad social de empresas, cooperativas o del Estado. Las consultas en servicios de salud públicos (que no necesariamente son gratuitos) generalmente ofrecen cuidados considerados como básicos, y su calidad depende de cada programa, los recursos y las leyes de cada país. Tratamientos de rehabilitación, ortodoncia y estéticos, con algunas excepciones, no son contemplados en estos servicios y las personas se ven forzadas a utilizar servicios privados para terminar con su plan de tratamiento, incluyendo rehabilitaciones simples y complejas. Los profesionales que practican la odontología en el sector privado negocian con la capacidad de pago de las personas y, en muchos casos, los profesionales practican su profesión en varios sectores sociales y combinan las consultas privada y pública.

La negociación entre profesional de la salud y paciente se basa en una transacción entre oferta de servicios y poder adquisitivo para acceder a ellos. De esta forma, la práctica odontológica reproduce desigualdades sociales de la población: los ciudadanos tienen acceso a servicios 
según su capacidad de pago. Círculos odontológicos parecen aceptar diferentes estándares de manejo para las personas según su condición socioeconómica. Un grupo de odontólogos, de diversos países, que discutió los aspectos éticos, de equidad y la responsabilidad de la profesión, comentó que las mejores prácticas (el tratamiento ideal) puede ser un factor de confusión en las escuelas de odontología, ya que hablar de tratamientos ideales parece implicar que "...bajo cualquier circunstancia, un cierto tipo de 'prácticas' siempre serán las mejores. En un mundo con unas variaciones enormes en características económicas, ambientales, sociales y culturales tal concepto es difícilmente realizable. En cambio, se considera que la lista de 'mejores prácticas' hace referencia más a las metas hacia las cuales esperamos llegar, si todos los otros factores son iguales(19)".

Los factores que deben tenerse en cuenta para la equidad en salud oral, según los mismos autores, son los aspectos socioeconómicos, culturales, étnicos, de género, de religión, de lenguaje y discapacidad. Claramente, hay una diferencia con la medicina, ya que se favorece el análisis individual y no los protocolos universales. Parece ser que los protocolos se reconocen como las "mejores prácticas" o el "tratamiento ideal", pero se acepta que esto sólo es para unos pocos que cumplen con determinadas características. Si bien podemos estar de acuerdo en que esta propuesta relativista de la odontología puede ser útil frente a parámetros culturales y religiosos (aunque es difícil saber cómo los autores diferencian los dos aspectos interrelacionados), aspectos de género (pensando en que algunos tratamientos pueden ser modificados en caso de embarazo), raciales (tratamientos odontológicos deben tener en cuenta la raza en la transformación de la apariencia de la sonrisa y el rostro, por ejemplo) y la discapacidad (no tanto porque a los discapacitados no se les puedan ofrecer los mejores tratamientos, sino porque ciertas limitaciones físicas pueden favorecer ciertos tipos de tratamientos que requieren cuidados más simples), no es muy satisfactorio un abordaje relativista cuando se plantea que diferencias en condición socioeconómica generan prácticas diferentes. Pareciera que con esta postura la profesión odontológica acepta que sus prácticas tienen que seguir un quehacer liberal, ligado al mercado y a la capacidad de pago individual, y, por tanto, no existe un planteamiento ético sobre las necesidades en salud oral de la población que vive en pobreza o no puede pagar los tratamientos ideales.

En los consultorios privados, los odontólogos, rápidamente y en su proceso de balancear oferta de servicios con capacidad de pago de los pacientes, producen una lista de servicios que van desde los ideales o adecuados hasta los cuidados mínimos o emergenciales, pasando por las múltiples combinaciones que incluyen medidas que buscan llegar lo más cercanamente posible al tratamiento ideal. El plan de tratamiento se organiza, generalmente, en cuatro acciones:

1) Urgencias: acciones que se relacionan, generalmente, con episodios de dolor.

2) Urgentes: acciones que necesitan atención a un corto plazo y que, generalmente, incluyen la eliminación de focos infecciosos.

3) Rehabilitación simple: medidas que ameritan atención en un plazo entre corto y medio y que dependen de la capacidad de pago de la persona. Si la persona puede pagar por estos tratamientos (por ejemplo, restauraciones), la velocidad de éstos se determina también por la prontitud con la cual el paciente pueda hacer los pagos.

4) Rehabilitación compleja: medidas de largo plazo que dependen mucho más de la capa- 
cidad de pago (por ejemplo, incrustaciones, prótesis o implantes).

Otros tratamientos pueden ubicarse en la transición de varias categorías. Por ejemplo, los tratamientos de conductos se pueden iniciar como una urgencia, son evidentemente urgentes, pero la posibilidad de acabar con el procedimiento, debido a sus altos costos, hace que se demore y siga una relación tiempo/capacidad de pago, similar a la rehabilitación simple. $\mathrm{Si}$ el tratamiento de conductos exige una rehabilitación más compleja, la posibilidad de terminarlo y llegar al procedimiento ideal incluye la negociación interna del paciente con su presupuesto, para ver cómo puede pagar, y con el profesional para obtener descuentos y facilidades de pago.

Las leyes del mercado y el enfoque liberal de la odontología hacen que la práctica odontológica se asemeje a otros bienes de consumo y son frecuentes las negociaciones alrededor del descuento y los atrasos en los pagos. Para cumplir con el plan de tratamiento se exige a los pacientes pagos mínimos y, cuando el paciente no puede pagar sus cuotas, éste se aplaza y comienza una nueva negociación para terminarlo y concluir los pagos. En estas negociaciones, varios profesionales sienten que su trabajo no es valorado por los pacientes o la comunidad y, cuando deciden trabajar con sectores de bajos recursos económicos, las actividades terapéuticas que pueden desarrollar son limitadas. En sectores pobres el tipo de tratamientos se limita, por lo general, a las urgencias y los urgentes. El ideal se reemplaza por procedimientos más agresivos (por ejemplo, extracciones) y se deja de lado la posibilidad de hacer intervenciones más complejas y rehabilitaciones. La negociación entre profesional y paciente es difícil ya que, por un lado, aquél es conciente de las limitaciones económicas de quienes asisten a su consulta, pero sabe que las necesidades en salud sobrepasan lo que las personas pueden pagar y, por el otro, los profesionales también tienen que cumplir con sus obligaciones económicas y administrativas y se ven obligados a reducir o abandonar los tratamientos subsidiados o gratuitos.

Adicionalmente, grandes empresas con ánimo de lucro se están apropiando de la prestación de servicios de salud oral privada y se observa una competencia de precios y servicios con los consultorios particulares. La "calidad" y el "costo" son las variables utilizadas en las campañas de mercadeo y publicidad de las grandes empresas, las cuales son generalmente suficientes para desterrar a los consultorios particulares de la región. Como resultado de este nuevo tipo de práctica de empresas, los odontólogos han visto un cambio en su condición laboral de trabajadores independientes a trabajadores subcontratados, generalmente bajo la modalidad de porcentaje. Si existe sobreoferta de odontólogos, debido a una falta de planeación y regulación estatal frente a las escuelas de formación de profesionales en salud, las empresas aprovechan las leyes del mercado en la contratación. En algunos países, los profesionales han visto que los salarios disminuyen constantemente y las condiciones laborales empeoran (mayores jornadas, equipos y materiales deficientes y menor pago). En estos casos, los odontólogos también pierden la libertad de decidir sobre los mejores tratamientos que el paciente necesita, ya que las limitaciones económicas del paciente y los intereses económicos de las empresas se traducen en una práctica en la cual los servicios ofrecidos (diagnósticos y terapéuticos) no son los ideales, sino los más rentables.

Entonces, los tratamientos odontológicos incluyen desde opciones ideales (las que son adecuadas según los parámetros técnicos y que incluyen generalmente las opciones más cos- 
tosas) hasta opciones mínimas, que se sabe no cumplen con los parámetros de tratamiento ideal. En este proceso de diferentes planes, en el que compiten los intereses de la industria de materiales dentales, las empresas privadas con ánimo de lucro y las diferentes capacidades de pago del paciente, las desigualdades sociales se perpetúan y aumentan. Este gran dilema moral del acceso a tratamientos adecuados no recibe la atención que se merece y, al parecer, la profesión satisface sus necesidades de responder a la población sin recursos con propuestas de salud pública que se enfocan en promoción y prevención en salud oral.

\section{Prevención para los pobres y tratamiento para los ricos}

Estudios recientes muestran que la prevalencia de enfermedades orales es mayor en personas que viven en la pobreza. La Organización Mundial de la Salud (OMS) reconoce que la pobreza y las desigualdades sociales juegan un papel fundamental en la presencia de enfermedades orales y en la posibilidad de recibir tratamientos. Por ejemplo, países en vías de desarrollo muestran una incidencia mucho mayor de cáncer bucofaríngeo, y el Noma, asociado a desnutrición, es casi exclusivo de África y el Sudeste asiático, las regiones más pobres del mundo(20). En Occidente, la caries y la enfermedad periodontal parecen ser un buen predictor de la pobreza en los niños y adultos, ya que el alto consumo de azúcares refinados y medidas higiénicas deficientes son más comunes en la población pobre $(20,21)$. Un estudio realizado en Nueva Zelandia, y que siguió a un grupo de niños por 26 años, encontró que las enfermedades orales no sólo eran más prevalentes en los niños de sectores más pobres, sino que, cuando adultos, ellos presentaban mayor presencia de enfermedades cardiovasculares y menor desarrollo físico y continuaban presentando mayor presencia de enfermedades orales (caries, enfermedad periodontal y sangrado gingival)(21). Otro hallazgo interesante de este estudio fue que, inclusive, si en el transcurso de la vida las personas mejoran su situación socioeconómica, adultos que fueron pobres cuando niños continúan presentando una mayor prevalencia de enfermedad periodontal y caries comparados con aquellos que nunca fueron pobres, lo cual indica que la relación entre la pobreza, salud oral e infancia determina en gran parte la salud y calidad de vida de los adultos(21).

¿Cuál ha sido el planteamiento político de la profesión odontológica frente a las desigualdades sociales? Desde las escuelas de odontología se ve claramente la disyuntiva. Los departamentos de salud pública y comunitaria (que al igual que en medicina son marginados y reciben menor atención que los departamentos clínicos) se dedican a enseñar acciones de promoción y prevención, generalmente mediante propuestas que incluyen la fluoración de la sal, agua, o pastas dentífricas, las campañas educativas y los sellantes. Los departamentos clínicos enseñan todas las actividades terapéuticas y enfatizan un plan de tratamiento que sigue las necesidades biológicas orales (como ya fue planteado: urgencias, urgentes, rehabilitación simple y rehabilitación compleja que dependen de la capacidad de pago de la persona y de las redes privadas o de seguridad social en salud de los países). Por otro lado, los departamentos de investigación en ciencias básicas biológicas, cuando existen, ven su quehacer investigativo coartado por los intereses de las empresas de materiales dentales y por el énfasis creciente en hacer que los centros educativos (privados y públicos) sólo realicen investigaciones rentables económicamente, lo cual se mide mediante la creación de productos patentables para uso y explotación comercial. Todo esto sin mencionar la falta de estrategias para integrar las investigaciones clínicas con 
las realizadas en ciencias básicas biológicas y sociales.

En esta práctica, basada en los mercados y que privilegia la atención clínica, los círculos de profesionales discuten que los odontólogos más exitosos no son necesariamente aquellos con más conocimientos o los que logran reducir índices de morbilidad oral en la población, sino los competentes en la práctica privada y buenos comerciantes y administradores (algunos de ellos administrando las nuevas empresas en salud). Los considerados como menos exitosos, desde el punto de vista económico, generalmente se reconocen como personas que tienen dificultades relacionándose con las leyes del mercado y se describen asimismo como profesionales que no saben cobrar. Profesionales que dedican sus carreras al servicio de poblaciones pobres, cuestionan en su práctica cotidiana la falta de acceso de la mayoría de la población a servicios integrales y adecuados de promoción, prevención, tratamiento y rehabilitación, sufren de dilemas morales frente a la ausencia de recursos propios y de sus pacientes, y se enfrentan, como pueden, a las leyes de mercado en salud.

\section{Propuestas de la salud oral para la salud pública: límites del flúor y de los programas educativos}

La Organización Panamericana de la Salud (OPS) ha participado en actividades preventivas de salud oral de la región, principalmente impulsando el programa de fluoración de la sal, las cuales han reducido de forma importante la morbimortalidad oral en los países que lo han implementado(22). La fluoración del agua ha sido reconocida como una de las medidas de salud pública más importantes en la historia, no sólo porque ha conseguido disminuir de forma importante la presencia de caries en la población, sino porque asegura que sus efectos se ex- tiendan a toda la población y, por tanto, es una medida efectiva para contrarrestar los vínculos entre desigualdades sociales y salud oral. Si bien la fluoración del agua disminuye desigualdades, la región latinoamericana debe tener en cuenta que municipios más pobres y menos desarrollados pueden no recibir agua fluorada por falta de desarrollo(23) y que aún se debate el riesgo/beneficio entre el flúor como agente protector de caries y como agente causante de fluorosis oral, esquelética y sistémica. Además, es evidente que, inclusive con medidas de flúor, la población aún sufre un elevado índice de morbilidad y mortalidad por las dos enfermedades orales más comunes, caries y enfermedad periodontal, las cuales son previsibles.

Recientemente se reconocen causas ambientales, económicas y sociales en la presencia de enfermedades orales $(19,24)$; sin embargo, el flúor y los aspectos conductuales se siguen considerando como los más importantes cuando se habla de promoción y prevención en salud oral. El enfoque en los comportamientos individuales hace que el complejo biosocial que determina la presencia de caries y enfermedad periodontal en las poblaciones se vea reducido de forma inapropiada a actividades educativas sobre higiene y dieta, desconociendo que higiene y dieta son dos procesos profundamente sociales. Sin restar importancia a la educación como estrategia en prevención, la literatura odontológica carece de definiciones sobre cómo los factores sociales influyen en el comportamiento humano, en los conocimientos, actitudes y prácticas en salud, o sobre cuáles son los límites de la educación y de otras actividades y programas preventivos. Por tanto, no se han planteado alternativas sobre cómo contrarrestar los efectos de los factores sociales en las enfermedades orales.

La literatura odontológica sobre factores de riesgo y su énfasis en los comportamientos in- 
dividuales más que en las condiciones sociales podría beneficiarse de nuevas teorías en medicina social y enfermedades infecciosas, que reconocen que la vulnerabilidad de personas y grupos poblacionales a éstas dista mucho de ser un factor individual exclusivamente y sí se trata de una compleja relación entre factores individuales, institucionales y sociales(25). En esta nueva línea teórica se entiende que cuando se enfatiza la responsabilidad individual de forma exagerada se termina culpando a las víctimas de sus enfermedades(26). La literatura en salud oral sigue comentando sobre los factores de riesgo individual, los hábitos alimenticios y medidas de higiene, e insiste en que cambios de mentalidad mediante educación van a resultar en éxitos preventivos(20). Tal estrategia se ha mostrado históricamente muy poco efectiva y las nuevas teorías sociales nos permiten entender que educación sin condiciones que garanticen que lo aprendido pueda ser llevado a la práctica no alcanza sus objetivos.

\section{Dos mitos de la odontología: los tratamientos odontológicos son costosos y prevención $\mathrm{v} / \mathrm{s}$ tratamiento}

La estrategia global para la salud oral de la OMS plantea que los tratamientos tradicionales para las enfermedades orales son muy costosos en los países industrializados e imposibles en la mayoría de países de bajos y medianos ingresos. Frente a los costos en aumento y los recursos limitados, la OMS insiste en la prevención y el control de las enfermedades orales(20). Sin embargo, no existe un análisis de costos adecuado que permita analizar cómo se distribuye el lucro generado en la consulta odontológica y si, de verdad, es imposible ofrecer tratamientos odontológicos adecuados a toda la población. Si hacemos un paralelo con las nuevas investigaciones en medicina social que apuntan al lucro excesivo y la influencia de la industria farmacéutica en la práctica clí- nica, podríamos pensar que otro tanto ocurre con las compañías multinacionales de materiales dentales en odontología y que los costos reales de los tratamientos odontológicos son desconocidos, ya que dependen de tales leyes del mercado.

La nueva meta de la estrategia regional de la OPS incluye servicios de salud oral eficaces en función del costo, destinados a eliminar las disparidades en el tratamiento y aumentar el acceso de la población a los servicios(22). Sin embargo, no es muy claro cuál es la estrategia planteada, ya que se ha mostrado que ciertas propuestas en salud pública, basadas en análisis "costo-beneficio-efectividad", no son adecuadas, porque éstas siguen los intereses de los que manejan los mercados de salud para asegurar el mayor margen de lucro posible, no responden a las necesidades en salud de la población y, en vez de aumentar, reducen el acceso a los tratamientos de las poblaciones pobres(3). Una discusión parecida ocurrió con ocasión de la epidemia del SIDA, de la cual la salud oral podría aprender mucho.

El Banco Mundial, en su informe ya citado(6), señaló que las terapias para el SIDA eran muy costosas para países en vías de desarrollo y recomendó centrar recursos y esfuerzos únicamente en prevención, ya que se consideraba más costo-efectivo. Brasil, contrariando esta propuesta, decidió ofrecer tratamiento universal y gratuito a todas las personas que lo necesitaban y mostró, con el tiempo, que no solamente los esfuerzos preventivos se favorecen con el tratamiento, sino que ofrecer procedimientos adecuados hace que las personas sufran de menos enfermedades y menos complicaciones y, por tanto, los sistemas de salud pueden ahorrar costos(27). Mientras el Banco Mundial estimó que Brasil iba a tener 1,2 millones de personas infectadas en el cambio de siglo, a finales de 2001 Brasil, integrando pre- 
vención con tratamiento, sólo contaba con 600.000 brasileños infectados, mostrando una reducción del $50 \%$ en las estimaciones previstas y un control efectivo de la epidemia $(28,29)$.

En cuanto al costo de los tratamientos, a pesar de que la terapia antirretroviral efectiva es muy costosa (US\$ 10.000 por paciente por año en países desarrollados), una serie de estrategias permitió a Brasil disminuir los costos de estas medicinas (a menos de US\$2.300 por paciente por año). Por un lado, el país comenzó a fabricar versiones genéricas de medicinas cuyas patentes habían expirado y logró disminuir los precios significativamente. Para las medicinas más nuevas, aún protegidas mediante leyes comerciales internacionales de patentes, el país logró reducciones significativas de precios al centralizar las compras y negociar rebajas sustanciales con las compañías dueñas de las patentes $(28,29)$. Además de estos ahorros, Brasil mostró que el acceso universal y gratuito a los tratamientos hizo que miles de brasileños mejoraran su calidad de vida y el país estima que, entre 1997 y 2001, se ahorraron más de mil millones de dólares al evitar hospitalizaciones y tratamientos más complejos(27).

Un enfoque similar podría aplicarse en la odontología y deslegitimar dos tabúes afianzados en la práctica de la profesión y en las políticas públicas. Los tratamientos odontológicos no deben ser vistos como costosos y la prevención debe siempre ir ligada al tratamiento. $\mathrm{Si}$ todas las personas tuviesen acceso a medidas preventivas y a tratamientos adecuados y oportunos, se disminuiría la presencia de enfermedades orales, se evitaría su avance y la aparición de complicaciones más graves y debilitantes, y se disminuiría la necesidad de tratamientos más costosos. Esto es mucho más relevante para el caso de la salud oral, no sólo porque las enfermedades orales más comunes son previsibles, sino porque tratamientos opor- tunos son mucho más fáciles, controlan y eliminan la enfermedad y evitan complicaciones más graves y tratamientos más complejos.

\section{Los mercados y la práctica odontológica}

La odontología surgió como rama de la medicina y en algunos países aún es una especialidad de ésta. A pesar de que la odontología se ha caracterizado por ser una profesión liberal, y talvez sea ésta la principal característica que lleva a muchas vocaciones, los procesos de institucionalización que se vivieron en la medicina también afectaron a algunas prácticas odontológicas (principalmente en el sector público y de seguridad social y, más recientemente, entre empresas de salud con ánimo de lucro que subcontratan a los odontólogos para que presten servicios). En este panorama de mercados en el cual la odontología está inmersa, los profesionales han visto cómo su práctica dejó de ser liberal y su calidad de vida ha empeorado. Por otro lado, la población sigue sin acceso adecuado a servicios de salud oral.

Con esta revisión muy rápida, espero que sea posible observar la manera como la odontología refleja las leyes del mercado y fomenta y afianza las desigualdades sociales. Espero que también haya señalado de forma convincente que la discusión sobre el papel de la odontología en la región latinoamericana es una discusión política que tiene que ver con redefinir (o definir) la profesión y las estrategias planteadas para responder a las necesidades de salud oral. Si bien es importante seguir avanzando en la producción de tratamientos de alta tecnología, la profesión necesita mucho más de medidas, programas y políticas altamente avanzadas para mejorar las condiciones precarias de salud oral de la mayoría de la población.

El reto ético más importante de la profesión odontológica es posicionarse políticamente, 
bien sea como profesión de la salud que sigue los principios de la medicina social o continuar como práctica que depende de las leyes del mercado. Organismos de salud internacionales parecen enfatizar el enfoque liberal e individualista de la salud y desconocen otras opciones como aquellas propuestas por la tradición de la medicina social. La OMS sigue insistiendo en que la caries dental y la enfermedad periodontal se pueden controlar y prevenir mediante la combinación de acciones comunitarias, profesionales e individuales que enfaticen la educación y los cambios de comportamiento(20). En cuestión de políticas, la OMS ha insistido, desde 1994, en que una medida de salud pública de interés para los países puede ser la exención de impuestos y aranceles que se aplican a ciertos productos cosméticos como son las pastas dentífricas con flúor para que a las personas les resulte más fácil comprarlas $(20,24)$. Tal recomendación es muy sospechosa, ¿por qué un organismo internacional de salud sugiere políticas públicas que benefician a ciertas compañías multinacionales de cosméticos?

Ante la falta de pastas dentífricas con flúor, dado el caso que esta estrategia sea más adecuada o complementaria a la fluoración del agua o la sal, organismos internacionales en salud pueden sugerir diferentes estrategias, como producción local o regional de pastas dentífricas (un proceso muy simple y económico) o suministro gratuito y universal de pastas. El ejemplo de las estrategias brasileñas frente al SIDA es evidencia de que estas dos alternativas (la producción local y la distribución gratuita) son mucho más efectivas que la sumisión al poder económico de las compañías multinacionales y el desconocimiento de los impedimentos económicos causados por la pobreza.

La recomendación de la OMS frente a las pastas dentífricas con flúor es mucho más sospechosa cuando en sus reportes se expresa, en forma elocuente, que dos factores principales son los responsables de la presencia de caries en la población: la falta de flúor y el alto consumo de azúcares refinados. Para el consumo de estos últimos, sin embargo, la OMS no tiene recomendaciones en salud pública similares a aquellas de los cosméticos, es decir, aumentar las medidas arancelarias y de impuestos a las bebidas con azúcares refinados. Por el contrario, la OMS propone que se implementen consejerías nutricionales para que las personas disminuyan su consumo de azúcares. El sesgo en la balanza es claro.

Recordemos que organismos financieros y grupos económicos han penetrado diferentes organismos de las Naciones Unidas, incluyendo a la OMS, y que las últimas políticas, análisis y recomendaciones de este organismo han sido criticados fuertemente por mostrar un viraje peligroso desde velar por la salud como derecho humano universal e integral a favorecer intereses particulares de compañías farmacéuticas, biotecnológicas y de $\operatorname{seguros}(11)$. Algo parecido ha sucedido en la odontología, por mucho tiempo, y estamos en la hora de comenzar a discutirlo y hacer algo al respecto.

\section{Referencias}

1. Comisión Económica para América Latina y el Caribe. Panorama Social de América Latina. Washington: ECLAC; 2004.

2. Waitzkin H, Iriart C, Estrada A, Lamadrid S. Social Medicine Then and Now: Lessons From Latin America. American Journal of Public Health 2001; 91(10): 1592-1601. 
3. Rylho-Bauer B, Farmer P. Managed Care or Managed Inequality? A Call for Critiques of Market-Based Medicine. Medical Anthropology Quaterly 2002; 16(4): 476-502.

4. Almeida C. Reforma de sistemas de servicios de salud y equidad en América Latina y el Caribe: algunas lecciones de los años 80 y 90. Cadernos de Saúde Publica 2002; 18(4): 905-925.

5. Armada F, Muntaner C. The Visible Fist of the Market: Health Reforms in Latin America. In: Castro A, Singer M, (eds.) Unhealthy Health Policy. A Critical Anthropological Examination. Walnut Creek: Altamira Press; 2004: 29-42.

6. The World Bank. World Development Report 1993. Investing in Health. New York: Oxford University Press: 1993.

7. Iriart C, Merhy EE, Waitzkin H. Managed Care in Latin America: the new common sense in health policy reform. Social Science \& Medicine 2001; 52: 1243-1253.

8. Stocker K, Waitzkin H, Iriart C. The Exportation of Managed Care to Latin America. New England Journal of Medicine 1999; 340(14): 1131-1136.

9. Good MJD. American Medicine: The Quest for Competence. Berkeley and Los Angeles. London: University of California Press: 1995.

10. Schraiber LB. O Médico e seu Trabalho. Límites da Liberdade. São Paulo: Hucitec; 1993.

11. Navarro V. The world situation and the WHO. The Lancet 2004; 363: 1321-1323.

12. Pellegrino ED. The Commodification of Medical and Health Care: The Moral Consequences of a Paradigm Shift from a Professional to a Market Ethic. Journal of Medicine and Philosophy 1999; 24(3): 243-266.

13. Smith R. Medical journals are an extension of the marketing arm of pharmaceutical companies. $P$ Los Med 2005; 2(5): e138.

14. Weiss L. Private Medicine and Public Health: Profits, Politics, and Prejudice in the American Health Care Enterprise. Cumnor Hill: Westview Press: 1997.

15. Angell M. The truth about drug companies: How they deceive us and what to do about it. New York: Random House: 2005.

16. Smedley BD, Stith AY, Nelson AR, (eds.) Unequal Treatment. Confronting Racial and Ethnic Disparities in Health Care. Washington DC: Institute of Medicine. The National Academies Press: 2003.

17. Good MJD, James C, Good BJ, Becker AE. The Culture of Medicine and Racial. Ethnic and Class Disparities in Health Care. In B. D.

18. Gilbert G, Duncan R, Shelton B. Social determinants of tooth loss. Health Serv Res 2003; 38(6): 1843-1862.

19. Hobdel M, Sinkford J, Alexander C, et al. Ethics, equity and global responsibilities in oral health and disease. European Journal of Dental Education 2002; 6(3): 167-178.

20. Petersen PE. The World Oral Health Report 2003. Geneva: WHO: 2003.

21. Poulton R, Caspi A, Mine B, et al. Association between children's experience of socioeconomic disadvantage and adult health: a life course study. The Lancet 2002; 360: 1640-1645. 
Pobreza y desigualdades sociales - C. Abadía Barrero

22. OPS. El Progreso en la Salud de la Población. Informe Anual del Director. Washington: OPS; 2000.

23. Peres MA, Fernandes LS, Peres KG. Inequality of water fluoridation in Southern Brazil-the inverse equity hypothesis revisited. Social Science \& Medicine 2004; 58, 1181-1189.

24. Petersen PE, Lennon M. Effective use of fluorides for the prevention of dental caries in the 21 st century: the WHO approach. Community Dentistry and Oral Epidemiology 2004; 32: 319-321.

25. Farmer P. Infections and Inequalities: The Modern Plagues. Berkeley and Los Angeles: University of California Press, Ltd; 1999.

26. Castro A, Farmer P. El sida y la violencia estructural: La culpabilización de la víctima. Cuadernos de Antropología Social 2003; 17: 31-49.

27. Ministry of Health of Brazil. Response: The experience of the Brazilian AIDS Programme. Brasilia: Ministry of Health; 2002.

28. Piot P, Coll Seck AM. International response to the HIV/AIDS epidemic: planning for success. Bulletin of the World Health Organization 2001; 79: 1106-1112.

29. Reardon C. AIDS: How Brazil Turned the Tide. Can others emulate its success? Ford Foundation Report 2002; 33(3): 8-13.

Recibido: 03 de enero de 2006

Aceptado: 30 de enero de 2006 